\title{
Porcine mononuclear phagocyte subpopulations in the lung, blood and bone marrow: dynamics during inflammation induced by Actinobacillus pleuropneumoniae
}

\author{
Petra Ondrackova ${ }^{1}$, Katerina Nechvatalova ${ }^{1}$, Zdenka Kucerova ${ }^{1}$, Lenka Leva ${ }^{1}$, \\ Javier Dominguez ${ }^{2}$, Martin FALDYnA ${ }^{1 *}$ \\ ${ }^{1}$ Department of Immunology, Veterinary Research Institute, Hudcova 70, 62100 Brno, Czech Republic \\ ${ }^{2}$ Departmento de Biotecnologia, Instituto Nacional de Investigacion y Tecnologia Agraria y Alimentaria (INIA), \\ Madrid, Spain
}

(Received 7 October 2009; accepted 2 June 2010)

\begin{abstract}
Mononuclear phagocytes (MP) are cells of nonspecific immunity, playing an essential role in defense against bacterial pathogens. Although various MP subpopulations have been described in the pig, relations among these populations in vivo are unknown to date. The present study was aimed at describing porcine MP subpopulations infiltrating inflamed tissue of pigs under in vivo conditions. Actinobacillus pleuropneumoniae (APP) infection was used to induce an inflammatory response. CD172 $\alpha$, CD14, CD163, MHCII and CD203 $\alpha$ cell surface molecules were used to identify MP by flow cytometry. Changes in MP subpopulations in the peripheral blood (PB) and bone marrow (BM) compartments along with the analysis of MP appearing in the inflamed lungs were assessed to elucidate the possible origin and maturation stages of the infiltrating MP. The MP population migrating to the inflamed lungs was phenotype $\mathrm{CD} 14^{+} \mathrm{CD} 163^{+}$ $\mathrm{CD} 203 \alpha^{+/-} \mathrm{MHCII}^{+/-}$. Concomitantly, after APP infection there was an increase in the PB MP CD14 $\mathrm{CD} 163^{+} \mathrm{CD} 203 \alpha^{-} \mathrm{MHC} \mathrm{II}^{-}$population, suggesting that these cells give rise to inflammatory monocytes/ macrophages. The CD203 $\alpha$ and MHCII molecules appear on these cells after leaving the PB. In healthy animals, the BM MP precursors were represented by $\mathrm{CD} 14^{-} \mathrm{CD} 163^{-}$cells maturing directly into $\mathrm{CD} 14^{+}$ $\mathrm{CD}_{163^{-}}$that were then released into the $\mathrm{PB}$. After infection, an altered maturation pathway of MP precursors appeared, represented by $\mathrm{CD} 14^{-} \mathrm{CD} 63^{-} \mathrm{CD} 203 \alpha^{-} \mathrm{MHCII}^{-} \mathrm{MP}$ directly switching into $\mathrm{CD}^{+} 4^{+} \mathrm{CD} 163^{+} \mathrm{CD} 203 \alpha^{-} \mathrm{MHCII}^{-}$MP. In conclusion, two different MP maturation pathways were suggested in pigs. The use of these pathways differs under inflammatory and noninflammatory conditions.
\end{abstract}

mononuclear phagocyte / pig / Actinobacillus pleuropneumoniae

\section{INTRODUCTION}

Mononuclear phagocytes (MP) are cells of nonspecific immunity that play an essential role in the defense against bacterial pathogens.

The MP system is a highly heterogeneous cell population dispersed throughout the organism. Macrophages, the final developmental

\footnotetext{
*Corresponding author: faldyna@vri.cz
}

stage of the MP lineage, originate from a specific progenitor in the bone marrow (BM) that sequentially develops into monoblasts, promonocytes and monocytes [9]. Monocytes enter the blood circulation and from there they migrate to various tissues, thereafter undergoing further differentiation into macrophages.

It was long believed that monocytes comprise a homogeneous population of blood cells. Recent evidence, however, indicates that blood 
monocytes may consist of several subpopulations of cells differing by size, nuclear morphology, granularity, and functionality (reviewed by $[11,17])$. Based on the expression of cell surface molecules, two major subpopulations of blood monocytes have been described to date in humans, mice and rats.

The functional aspects of monocyte heterogeneity have been best described in mice. BM macrophage and dendritic cell progenitors give rise to $\mathrm{Ly}^{6} \mathrm{C}^{\text {hi }} \mathrm{BM}$ monocytes, which are sequentially released into the bloodstream [16]. After 3 days of circulation in the bloodstream, the Ly6C $\mathrm{C}^{\text {hi }}$ monocytes switch their phenotype and become Ly6C $\mathrm{C}^{\text {low }}$ blood monocytes [22]. Under steady state conditions, more mature Ly6C ${ }^{\text {low }}$ blood monocytes migrate to the tissue and renew the population of tissue-specific resident macrophages $[10,13]$. Under inflammatory conditions, the less mature Ly6C ${ }^{\text {hi }}$ blood monocyte subset is the only one that is able to migrate to the inflamed tissue [10].

Two distinct subpopulations of blood monocytes have been described in pigs similarly to cases in humans, mice and rats. However, information about MP heterogeneity and their role in vivo remains scarce $[3,4]$. Differentiation is first based on the expression of the CD163 cell surface molecule [15]. CD163- monocytes were found to be smaller and less granular than $\mathrm{CD}_{163}{ }^{+}$. Moreover, small differences in the expression intensity of many other cell surface markers have been observed $[3,5,15]$. The differing ability of porcine monocyte subsets to produce TNF- $\alpha$, IL-1 $\beta$ and IL-10 suggest that functional differences between porcine monocyte subsets also exist $[5,15]$. The expression of CD163 on monocytes probably identifies a cell population in a more advanced maturation stage than that of $\mathrm{CD} 63^{-}$blood monocytes [15]. This is supported by the fact that CD163- monocytes could switch their phenotype to $\mathrm{CD} 163^{+}$after in vitro cultivation [5]. All the in vitro experiments suggest that $\mathrm{CD} 163^{+}$monocytes are a more mature form of blood monocytes and give rise to both tissue macrophages and dendritic cells. However, the in vivo function of less mature forms of porcine monocytes, which are believed to play an important role as precursors of inflammatory macrophages in mice and humans, is completely unknown in pigs at present. The aim of this work was to describe which developmental pathways used by porcine MP subpopulations are involved in infiltrating inflamed tissue under in vivo conditions. Various cell surface molecules that are known to be variably expressed by porcine MP subpopulations in vitro [5] were used for their identification. Moreover, the possible origin and maturation stages of these infiltrating MP were assessed in the PB and BM.

Actinobacillus pleuropneumoniae (APP) was chosen as the causative agent model for inducing an inflammatory response in lungs. APP is a Gram-negative, encapsulated bacterium that colonizes porcine lungs and causes pleuropneumonia. The bacteria bind to cells of the lower respiratory tract [2]. Clinical signs and pathological changes of the disease already appear within a few hours after experimental infection [1]. The infection of non-immunized pigs is followed by destruction of alveolar macrophages and rapid influx of professional phagocytes and lymphocytes to the tissue [1] and bronchoalveolar space [7, 8]. A rapid cellular influx of MP into infected lungs together with a specific localization of the pathogen in the lungs predetermine experimental APP infection to be an appropriate model for observing MP migration under inflammatory conditions in pigs.

\section{MATERIALS AND METHODS}

\subsection{Animals and experimental infection}

Ten 8-week-old healthy piglets with low levels of APP-specific antibodies were used in the experiment. The pigs were kept in the accredited barrier-type animal facilities of the Veterinary Research Institute. The animal care protocol for this experiment followed the Czech guidelines for animal experimentation and was approved by the Branch Commission for Animal Welfare of the Ministry of Agriculture of the Czech Republic. The piglets were allowed to acclimatize in the animal facilities for one week, and then the experimental infection was performed.

The infection with APP was performed intranasally during inhalation, and the infectious dose of $2 \times 10^{9}$ bacteria was administered to the second third of each nasal cavity. Health status was monitored 
during the entire experiment and clinical signs of respiratory disorders were recorded (increased dyspnoea, coughing, anorexia, depression and lethargy).

Seven piglets were infected with APP, while 3 piglets were left as noninfected controls. The three noninfected piglets were euthanized at infection time 0 . Infected piglets were euthanized $24 \mathrm{~h}$ (3 piglets) and $72 \mathrm{~h}$ (4 piglets) after infection.

\subsection{Blood and tissue sampling}

Immediately after euthanasia, samples of sternum and lung tissue were acquired. Simultaneously, heparinized blood samples from all living piglets were taken at infection time 0 (10 piglets) and then 6 (7 piglets), 24 (7 piglets), 48 (4 piglets) and 72 (4 piglets) $\mathrm{h}$ after infection.

\subsection{Processing of the samples}

Total white blood cell count was ascertained using an auto hematology analyzer (BC-2800Vet, Shenzhen Mindray Bio-Medical Electronics, Shenzhen, People's Republic of China). Red blood cells were lysed with ammonium chloride solution $\left(154.4 \mathrm{mM} \mathrm{NH} \mathrm{NH}_{4} \mathrm{Cl}\right.$, $10 \mathrm{mM} \mathrm{KHCO} 3,0.1 \mathrm{mM}$ EDTA, all from SigmaAldrich, St. Louis, USA), leukocyte suspension was then washed with cell washing solution (CWS, phosphate buffered saline containing $1.84 \mathrm{~g} / \mathrm{L}$ EDTA, $1 \mathrm{~g} / \mathrm{L}$ sodium azide and $4 \mathrm{~mL} / \mathrm{L}$ gelatin, all from Sigma-Aldrich) and the final peripheral blood leukocyte (PBL) count was ascertained.

The last sternebrum was disengaged from the connective tissue and periost and the BM cells were rinsed from the bone tissue with $100 \mathrm{~mL}$ of CWS using a $1.7 \mathrm{G}$ needle. Red blood cells were lysed with ammonium chloride solution, leukocyte suspension was washed with CWS, and the final bone marrow leukocyte (BML) count was ascertained.

During the isolation of lung MP, the lung tissue was repeatedly weighed in order to allow calculation of final MP count per gram of tissue (see Sect. 2.6). The caudal part of the cranial lobe of the left lung was separated, weighed, and the vasculature and main bronchus were cannulated with a $1.7 \mathrm{G}$ plastic intravenous catheter. The vasculature was washed with $80 \mathrm{~mL}$ of Dulbecco's phosphate buffered saline (Sigma-Aldrich) containing $0.2 \%$ EDTA to remove blood cells from the vasculature. Then, the bronchoalveolar space was lavaged three times with $50 \mathrm{~mL}$ of CWS. The alveolar leukocytes (AL) thus obtained were washed two times with CWS and counted. The entire lavaged lobe was weighed. A piece of the lobe tissue was cut, weighed, disintegrated using a fine nylon mesh, and washed with CWS. The remaining red blood cells were then lysed with ammonium chloride solution. Finally, the interstitial leukocytes (ISL) obtained were washed again with CWS and counted.

Since typical APP lesions appeared in the cranial lobe of the right lung $72 \mathrm{~h}$ after infection, an analysis of ISL within the lesion (lesion leukocytes, LL) was also performed. A piece of the lesion was excised, disintegrated using a fine nylon mesh, washed with CWS, and LL were counted.

\subsection{Detection of cell surface molecules by flow cytometry}

Staining of cell surface molecules was performed as described previously [24], with small modifications. Various setups of cell surface molecule combinations, including a list of all primary and secondary antibodies used in the experiment are shown in Tables I and II.

The $5 \times 10^{5}$ of BML, PBL, AL, ISL or LL were mixed with $20 \mu \mathrm{L}$ of primary antibody cocktail (see Tab. II, setups nos. 1-5) and $20 \mu \mathrm{L}$ of heat-inactivated goat serum, then incubated for $20 \mathrm{~min}$ at $4{ }^{\circ} \mathrm{C}$. The cells were rinsed twice with CWS. Then, $50 \mu \mathrm{L}$ of secondary antibody cocktail (anti-IgG2b: FITC, anti-IgM: PE and anti-IgG1: AlexaFluor647) was added and the cells were incubated for another $20 \mathrm{~min}$ at $4{ }^{\circ} \mathrm{C}$. Finally, propidium iodide (Sigma-Aldrich) was added to each tube and the samples were measured as soon as possible using FACSCalibur flow cytometer (Becton-Dickinson, New Jersey, USA). At least 50000 events were acquired.

Since two distinct clones of anti-CD172 $\alpha$ antibody (74-22-15 and DH59B) were used for detecting this molecule, it was verified during preliminary experiments by two-color analysis that both clones have identical expression profile.

The post-acquisition analysis of data was performed using the Summit software (DAKO, Glostrup, Denmark).

\subsection{Gating strategy}

The staining of cell surface molecule combinations listed in Table II was performed in lung (setups nos. 1-3 and 5), PB and BM leukocytes (setups nos. 1-5).

The gating strategy for lung monocytes/macrophages is shown in Figure 1. MP were identified as viable $\mathrm{CD} 172 \alpha^{+} \mathrm{SWC}^{-}$mononuclear leukocytes. 
Table I. Primary mouse anti-porcine cell surface marker antibodies used in the experiment.

\begin{tabular}{lccccc}
\hline Antibody & Clone & Subisotype & Producer & Catalogue & Expression profile \\
\hline CD14 & MIL-2 & IgG2b & Serotec & MCA1218 & $\mathrm{mo} / \mathrm{mf}$ neu \\
CD163 & 2A10/11 & IgG1 & Serotec & MCA2311 & subset of mo/mf, DC \\
CD172 $\alpha$ (SWC3) & DH59B & IgG1 & VMRD & DH59B & $\mathrm{mo} / \mathrm{mf}$, neu, eo, DC \\
CD172 $\alpha$ (SWC3) & 74-22-15A & IgG2b & JL & - & $\mathrm{mo} / \mathrm{mf}$, neu, eo, DC \\
CD203 $\alpha$ (SWC9) & PM19-7 & IgG1 & Serotec & MCA1973 & mf \\
SWC8 & MIL-3 & IgM & JL & - & neu, eo, subset of ly \\
MHCII (SLA-DQ) & K274.3G8 & IgG1 & JL & - & subset of mo, mf, ly \\
\hline
\end{tabular}

JL: a generous gift from Dr J.K. Lunney (Animal Parasitology Institute, Beltsville, MO, USA), originally produced by the University of Bristol. DC: dendritic cells; eo: eosinophils; ly: lymphocytes; mo: monocytes; mf: macrophages; neu: neutrophils.

Table II. Setup of four-color staining with appropriate secondary fluorochrome-conjugated antibodies.

\begin{tabular}{lcccc}
\hline Setup No. & FL1 & FL2 & FL3 & FL4 \\
\hline 1 & CD14 & SWC8 & Propidium iodide & CD172 $\alpha$ (DH59B) \\
2 & CD172 $\alpha(74-22-15 \mathrm{~A})$ & SWC8 & Propidium iodide & CD163 \\
3 & CD172 $\alpha(74-22-15 \mathrm{~A})$ & SWC8 & Propidium iodide & MHCI \\
4 & CD14 & SWC8 & Propidium iodide & CD163 \\
5 & CD172 $\alpha(74-22-15 \mathrm{~A})$ & SWC8 & Propidium iodide & CD203 $\alpha$ \\
\hline
\end{tabular}

FL1: goat anti-mouse IgG2b: FITC, SouthernBiotech. FL2: goat anti-mouse IgM: PE, SouthernBiotech. FL4: goat anti-mouse IgG1: AlexaFluor647, Invitrogen.

Monocytes and macrophages were then differentiated according to their light scatter properties. The absolute counts of alveolar, interstitial and lesion $\mathrm{CD} 14^{+/-} \mathrm{CD}_{163^{+/-}} \mathrm{CD} 203 \alpha^{+/-}$or $\mathrm{MHCII}^{+/-}$monocytes or macrophages before and after APP infection were then calculated per gram of lung tissue.

The gating strategy for identifying particular PB and BM MP subpopulations is shown in Figure 2. Since CD203 $\alpha$ was expressed on neither PB nor BM monocytes, setup no. 5 (Tab. II) was not included in the further analysis.

\subsection{Calculation of the results}

Generally, the counts of MP subpopulations in the particular regions and gates were always expressed as proportions of these cells per 1000 leukocytes (after exclusion of cell debris). Final counts of particular cell subpopulations were then calculated and expressed as a proportion of 1000 leukocytes (BML) or they were further recalculated to $1 \mathrm{~mL}$ of blood (PBL). Since the weight of the whole lobe tissue before and after lavage, as well as the weight of the piece of disintegrated tissue, was ascertained during processing of the lung tissue, it was possible to express the numbers of AL, ISL and LL as absolute counts per $1 \mathrm{~g}$ of lung tissue.

\subsection{Statistical analysis of the results}

The differences between noninfected and infected animals were evaluated by the Mann-Whitney $U$ test (Prisma Software, Graph Pad Software Inc., San Diego, USA).

\section{RESULTS}

\subsection{Lung mononuclear phagocyte subpopulations}

The changes in macrophage count and phenotype in the lung compartments (AL and IS including LL) before and after APP infection were monitored. Since the blood monocytes are a source of lung macrophages during inflammation, the monocytes within the lung 


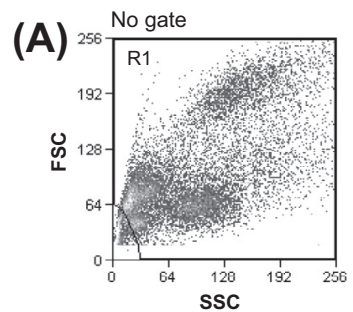

R1: all leukocytes

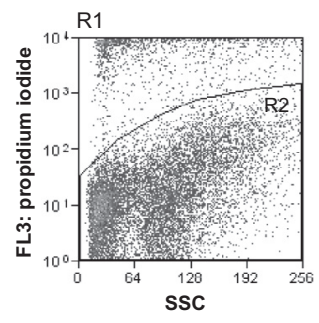

R2: viable

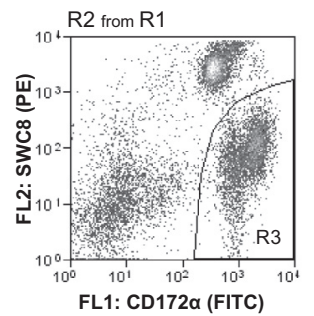

R3: monocytes + macrophages

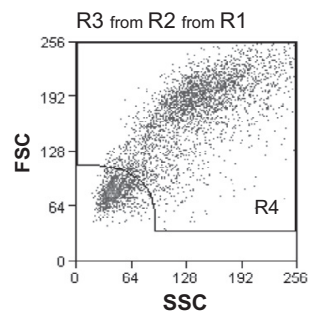

R4: macrophages not R4: monocytes
(B)

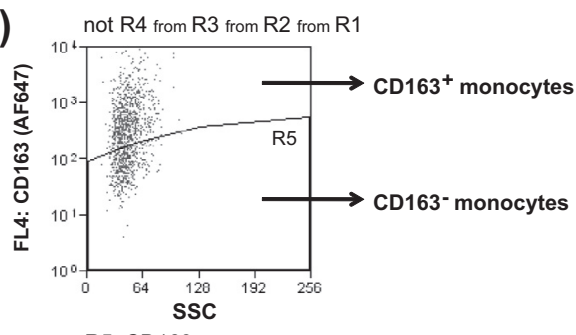

R5: CD163-

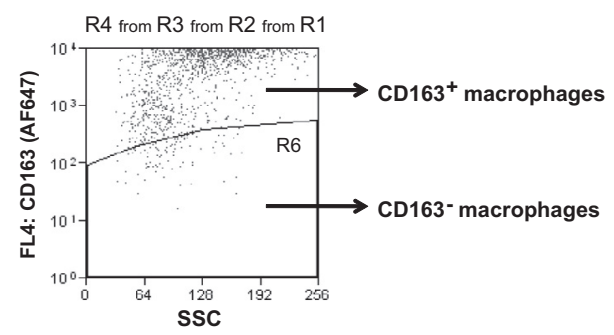

R6: CD163-

Figure 1. The general gating strategy for identification of particular monocyte/macrophage (M/M) subpopulations in the lung tissue. Setups nos. 1-3 and 5 from Table II were used for identification of M/M subpopulations. (A) General gating strategy for identification of viable $\mathrm{M} / \mathrm{M}$ in all setups. (B) Example of identifying $\mathrm{CD} 163^{+-} \mathrm{M} / \mathrm{M}$ in setup no. 2 .

compartments before and after infection were further evaluated.

\subsubsection{Lung macrophages}

In noninfected piglets, nearly all lung macrophages were $\mathrm{CD} 14^{+} \mathrm{CD}_{163^{+}} \mathrm{CD} 203 \alpha^{+}$and $\mathrm{MHCII}^{+}$.

The count and phenotypic profile of alveolar macrophages did not change significantly after infection (Fig. 3). The count of interstitial macrophages increased significantly, however, even though the phenotypic profile of most of these cells was similar after infection to that before infection $\left(\mathrm{CD}^{+} 4^{+} \mathrm{CD} 163^{+} \mathrm{CD} 203 \alpha^{+}\right.$and $\mathrm{MHCII}^{+}$). A small proportion of lesion macrophages were CD203 $\alpha^{-}$. Taken together, the count of lung interstitial macrophages increased during inflammation, and the most so within the lesion. The inflammatory lung macrophages had the same phenotypic profile as resident macrophages. The absence of the CD203 $\alpha$ molecule in some lesion macrophages suggests that the lesion macrophages originate from the monocytes, which typically lack this molecule.

\subsubsection{Lung monocytes}

In noninfected piglets, most lung monocytes were $\mathrm{CD} 14^{+} \mathrm{CD} 163^{+} \mathrm{CD} 203 \alpha^{+}$and $\mathrm{MHCII}^{+}$.

There appeared to be an increase in the number of AL monocytes and in the proportion of $\mathrm{CD} 203 \alpha^{-}$and $\mathrm{MHC} \mathrm{II}^{-}$subsets at $72 \mathrm{~h}$ postinfection, although these changes were not statistically significant (Fig. 3). The expression of CD14 and CD163 molecules did not change, and most of these AL monocytes were $\mathrm{CD} 14^{+}$ $\mathrm{CD}_{163}{ }^{+}$.

The count of IS monocytes increased significantly after infection. Similarly to macrophages, the most noticeable changes in monocyte count occurred in the LL monocytes. The newly recruited monocytes were largely $\mathrm{CD}_{14}^{+} \mathrm{CD} 163^{+} \mathrm{CD} 203 \alpha^{-} \mathrm{MHC} \mathrm{II}$. At $72 \mathrm{~h}$ post-infection, the majority of LL monocytes had the phenotype $\mathrm{CD} 14^{+} \mathrm{CD}_{163}{ }^{+} \mathrm{CD} 203 \alpha^{-}$ MHC $\mathrm{II}^{+}$. 


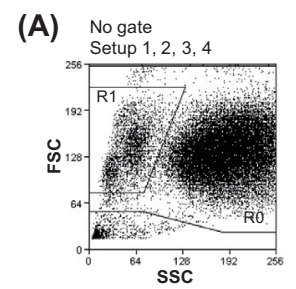

Ro: all leukocytes R1: MNL

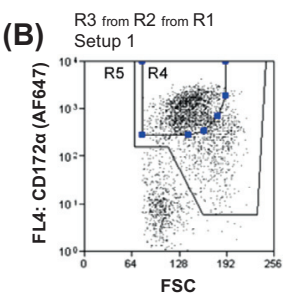

$$
\text { R4: CD172 } \alpha^{\text {hi }}
$$
R5: CD172a ${ }^{\text {lo }}$

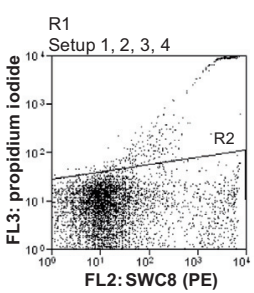

$\mathrm{R} 2$ : viable

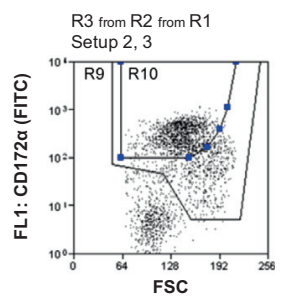

R10: CD172a ${ }^{\text {hi }}$ R9: CD172alo

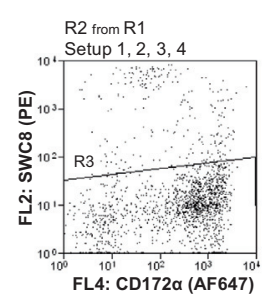

R3: SWC8-

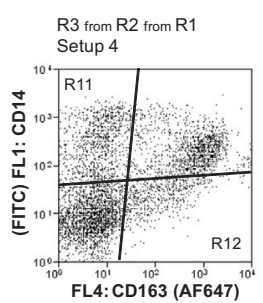

R11: CD14+

R12: CD163+
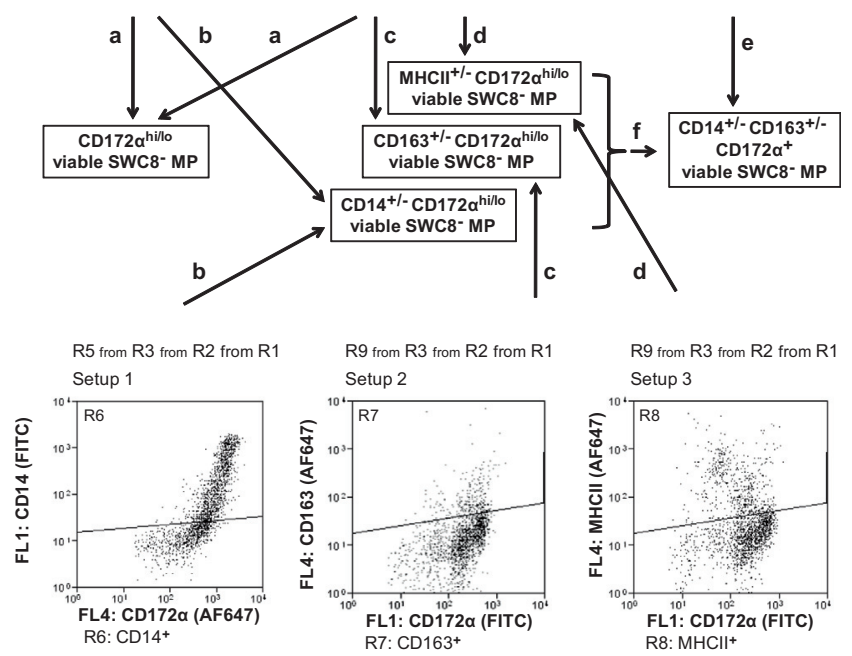

Figure 2. The general gating strategy for identification of particular mononuclear phagocyte (MP) subpopulations (bone marrow is shown). Setups nos. 1-4 from Table II were used for identifying MP subpopulations. (A) The counts of all acquired leukocytes (with exclusion of cells debris) were determined (R0). Then the general gating strategy for mononuclear leukocytes (MNL) was applied for all setups. This included gating of mononuclear (R1) viable (R2) leukocytes in which the potentially contaminating neutrophils (SWC8 ${ }^{+}$) were excluded (R3). (B) The CD172 $\alpha^{+}$(R5, R9) and CD172 $\alpha^{\text {hi }}$ (R4, R10) populations (a) were gated and CD172 $\alpha^{\text {hillo }}$ MP were calculated. The average from all setups, in which the CD172 $\alpha$ staining was performed (setups nos. 1-3) was finally expressed. The counts of CD172 $\alpha^{\text {hi/lo }}$ CD14 ${ }^{+/-}$ (b, setup no. 1, R4, R5, R6) or CD172 $\alpha^{\text {hi/lo }} \mathrm{CD} 163^{+/-}$(c, setup no. 2, R10, R9, R7) or CD172 $\alpha^{\text {hi/lo }} \mathrm{MHCII}^{+/-}$ (d, setup no. 3, R10, R9, R8) MP were ascertained. The $\mathrm{CD} 14^{+/-} \mathrm{CD} 163^{+/-}$MNL (e, setup no. 4, R11, R12) were gated and their count was ascertained. Using the known counts of CD172 $\alpha^{\text {hi/lo }} \mathrm{CD} 14^{+-}$and $\mathrm{CD} 172 \alpha^{\mathrm{hi} / \mathrm{lo}}$ $\mathrm{CD} 163^{+-}$(f), the counts of CD172 $\alpha^{+} \mathrm{CD} 14^{+/-} \mathrm{CD} 163^{+/-}$were finally calculated. (A color version of this figure is available online at www.vetres.org.) 


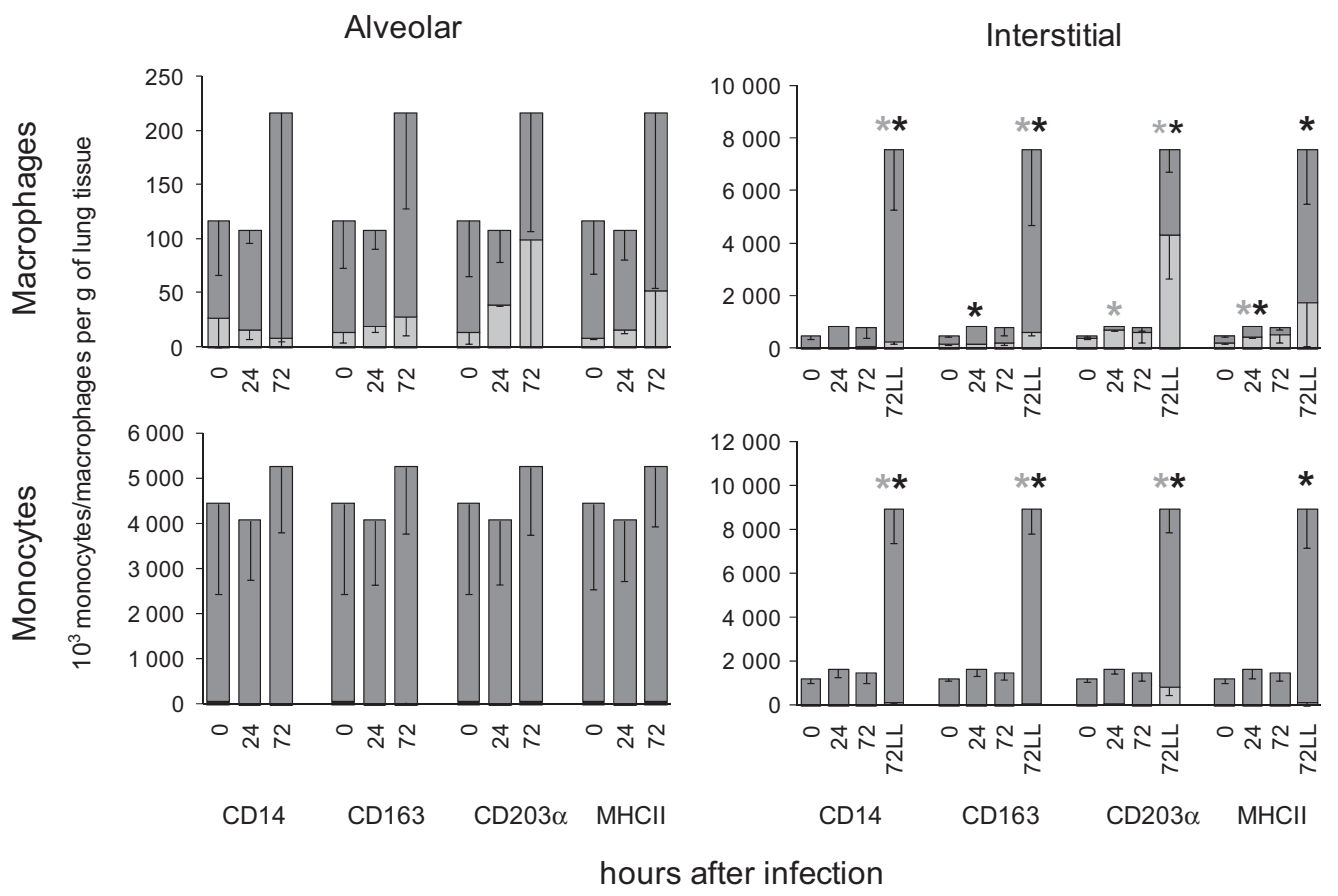

Figure 3. Monocyte/macrophage subpopulations within the lung tissue. Total counts were calculated of $\mathrm{CD}_{14}{ }^{+/-} \mathrm{CD}_{163}{ }^{+/-} \mathrm{CD} 203 \alpha^{+/-}$or $\mathrm{MHCII}^{+/-}$monocytes/macrophages (dark bar: $\mathrm{CD}^{+}$, clear bar: $\mathrm{CD}^{-}$) in alveolar and interstitial compartments, including the lesion (LL) compartment, before and 24 and $72 \mathrm{~h}$ after Actinobacillus pleuropneumoniae (APP) infection. The total counts of cells \pm SEM per gram of lung tissue are shown. The significant differences $(p<0.05)$ between noninfected $(0 \mathrm{~h}$ after infection) and infected piglets are marked with asterisks $(*)$. IS, interstitial.

This finding suggests that the newly recruited lung monocytes arise from blood monocytes since blood monocytes lack the CD203 $\alpha$ molecule, and moreover, most of them also lack the MHCII molecule. Regarding the fact that these recruited monocytes are $\mathrm{CD} 14^{+} \mathrm{CD}_{163}{ }^{+}$, it may be assumed that the population of blood monocytes with the phenotype $\mathrm{CD}^{+} 4^{+} \mathrm{CD}_{163}{ }^{+} \mathrm{CD} 203 \alpha^{-}$and $\mathrm{MHCII}^{+}$give rise to these newly recruited lung monocytes.

\subsection{Peripheral blood and bone marrow mononuclear phagocyte subpopulations}

In order to find the origin of lung inflammatory monocytes/macrophages, $\mathrm{PB}$ and BM MP were analyzed before and after infection.

\subsubsection{Peripheral blood mononuclear phagocyte subpopulations}

In noninfected piglets, two populations of PB MP were identified according to the intensity of CD172 $\alpha$ expression: CD172 $\alpha^{\text {hi }}$ and CD172 $\alpha^{\text {lo }}$ cells (Fig. 4A). Since CD172 $\alpha^{\text {hi }}$ cells were exclusively CD14 $4^{+}$and $\mathrm{CD} 172 \alpha^{\text {lo }}$ cells were $\mathrm{CD} 14^{-}$, they were further divided into monocytes (CD172 $\alpha^{\text {hi }} \mathrm{CD} 14^{+}$) and dendritic cells (CD172 $\alpha^{\text {lo }}$ CD14 ${ }^{-}$). The count of monocytes increased already $6 \mathrm{~h}$ after infection and remained elevated through the $72 \mathrm{~h}$ following infection (Fig. 4B). However, the count of dendritic cells did not change significantly during the entire course of the infection.

Then the CD14, CD163 and MHCII molecule expression by CD172 $\alpha^{\text {hi }}$ (monocytes) and $\mathrm{CD} 172 \alpha^{\text {lo }}$ (dendritic cells) PB MP was 
(A)

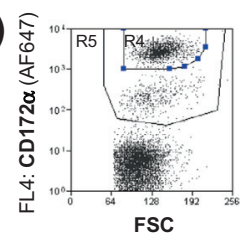

(C)

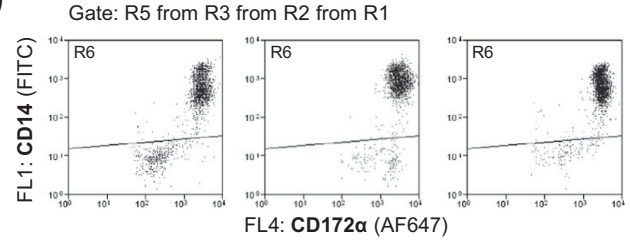

Gate: R9 from R3 from R2 from R1

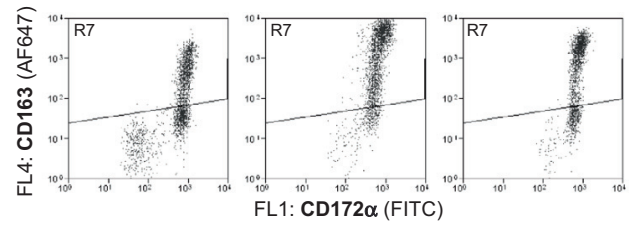

Gate: R9 from R3 from R2 from R1

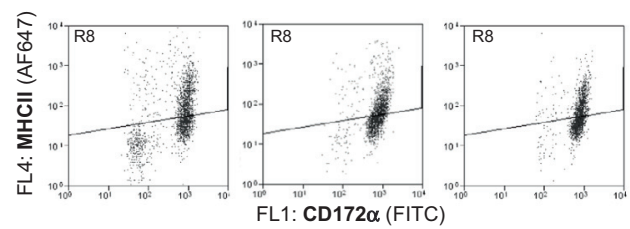

(E) Gate: R3 from R2 from R1

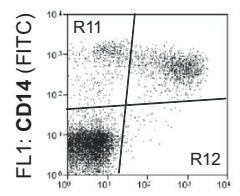

0

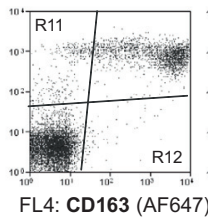

24

hours after infection
(B)

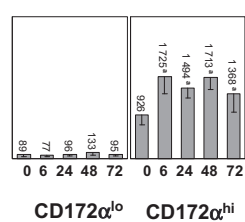

(D)

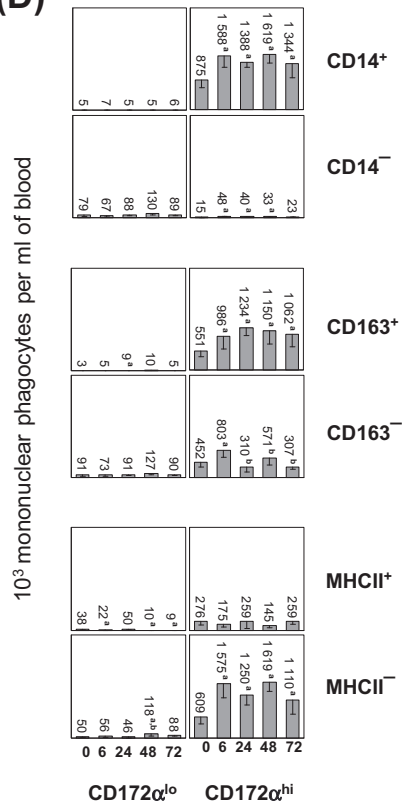

(F)

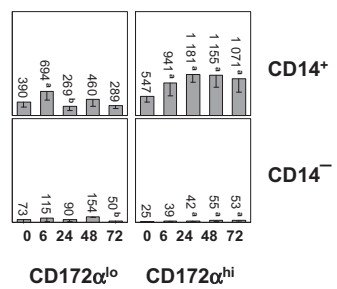

hours after infection

Figure 4. The cell surface molecule expression by peripheral blood mononuclear phagocytes (MP) after Actinobacillus pleuropneumoniae (APP) infection. The gating of MP subpopulations was performed as described in Figure 2. Representative dot-plots of CD172 $\alpha$ (A), CD14, CD163 or MHCII versus CD172 $\alpha$ (C) and CD14 versus CD163 (E) expression by MP are shown. The absolute counts of MP expressing CD172 $\alpha$ (B), CD14, CD163 or MHCII versus CD172 $\alpha$ (D) and CD14 versus CD163 (F) per mL of peripheral blood were calculated (data are mean \pm SEM). In the case of absolute counts of CD14 versus CD163 MP (F) the known counts of CD172 $\alpha^{\text {hi/lo }} \mathrm{CD} 14^{+/-}$and CD172 $\alpha^{\text {hi/lo }} \mathrm{CD} 163^{+/-}$(see Fig. 2F and Sect. 2), were used for calculations. Significant differences $(p<0.05)$ between noninfected $(0 \mathrm{~h}$ after infection) and APP-infected piglets are marked with "a". Significant differences $(p<0.05)$ between consecutive time-points are marked with " $b$ ". (A color version of this figure is available online at www.vetres.org.) 
analyzed (Figs. 4C and 4D). Nearly all (98\%) $\mathrm{CD} 172 \alpha^{\text {hi }}$ MP in noninfected piglets were $\mathrm{CD}_{14}{ }^{+}$. Fifty percent of them were $\mathrm{CD} 163^{+}$ and $31 \%$ of them were $\mathrm{MHCII}^{+}$.

The population of CD172 $\alpha^{\text {hi }} \mathrm{MP}$, which was found to be significantly elevated $6-72 \mathrm{~h}$ after infection, was almost exclusively of the $\mathrm{CD} 14^{+}$phenotype, although a small population of $\mathrm{CD} 14^{-}$cells was also transiently elevated 6-48 h after infection. As to the CD163 molecule, both $\mathrm{CD}_{163^{+}}$and $\mathrm{CD} 163^{-}$cells were elevated $6 \mathrm{~h}$ after infection. The CD163- cells returned to the initial count $24 \mathrm{~h}$ after infection, while the $\mathrm{CD} 163^{+}$cell count increased further. Their counts did not change further 48 and $72 \mathrm{~h}$ after infection. The elevated population of $\mathrm{CD} 172 \alpha^{\text {hi }} \mathrm{MP}$ was exclusively of the $\mathrm{MHCII}^{-}$phenotype.

Most CD172 $\alpha^{\text {lo }}$ MP in the noninfected piglets were $\mathrm{CD}^{-} 4^{-} \mathrm{CD} 163^{-} \mathrm{MHCII}^{+/}$. Although some significant changes in these populations were found after infection, these changes appeared to be random and were difficult to associate with the course of infection.

The results stated above suggest that the population of CD172 $\alpha^{\text {hi }}$ PB MP, which was elevated after infection, was of the $\mathrm{CD} 14^{+} \mathrm{CD} 163^{+}$ $\mathrm{MHCII}^{-}$phenotype. Moreover, there was a population of $\mathrm{CD}_{14}^{+} \mathrm{CD}^{-} 63^{-} \mathrm{MHCII}^{-} \mathrm{MP}$ which was transiently increased $6 \mathrm{~h}$ after infection.

This was not unambiguous, however, because the CD14, CD163 and MHCII molecules were always each stained separately, in combination with antibodies against the CD172 $\alpha$ molecules (setups nos. 1-3, Tab. II). To confirm the hypothesis, the staining of CD14/CD163 (setup no. 4, Tab. II) was performed. Since the CD172 $\alpha$ molecule could not be included in this setup, it was impossible to distinguish MP from lymphocytes during gating. However, because neither the CD14 nor CD163 molecule is expressed by lymphocytes, it was possible to calculate the real counts of CD14/CD163 MP populations using the values obtained above in setups nos. 1-3 (Fig. 4F). Thus, the CD14/CD163 expression by all mononuclear leukocytes before and after infection is depicted (Fig. 4E). It was shown that the population of CD172 $\alpha^{\text {hi }}$ PB MP which was transiently increased $6 \mathrm{~h}$ after infection was $\mathrm{CD}^{+} 4^{+} \mathrm{CD} 163^{-}$and the population which was increased during the whole course of infection was $\mathrm{CD} 14^{+} \mathrm{CD} 163^{+}$.

The population of $\mathrm{PB}$ dendritic cells $\left(\mathrm{CD} 172 \alpha^{\mathrm{lo}}\right)$ remained unchanged during the whole course of infection.

\subsubsection{Bone marrow mononuclear phagocyte subpopulations}

The exact identification of BM MP subpopulations was more complicated compared to that of PB due to the fact that the population of lymphoblasts can express the CD172 $\alpha$ molecule in amounts comparable to CD172 $\alpha^{\text {lo }} \mathrm{MP}$ [19]. Moreover, maturing BM MP show continuous expression of the $\mathrm{CD} 172 \alpha$ molecule (Fig. 5A) in contrast to PB MP where two separated populations of monocytes and dendritic cells were identifiable. Generally, compared to the $\mathrm{PB}$, much more heterogeneous MP populations exist in BM with respect to the expression of these cell surface molecules. Using the display of forward scatter against CD172 $\alpha$, however, it was possible to distinguish two populations of BM MP: CD172 $\alpha^{\text {hi }}$ $\mathrm{FSC}^{\mathrm{lo}}$ (more mature MP) and CD172 $\alpha^{\text {lo }} \mathrm{FSC}^{\text {hi }}$ (less mature MP) (Fig. 5A). The counts of both populations increased after infection (Fig. 5B).

Then, similarly to $\mathrm{PB}$, the expression of the CD14 CD163 and MHCII molecule by $\mathrm{CD} 172 \alpha^{\text {hi }}\left(\mathrm{FSC}^{\mathrm{lo}}\right)$ (more mature) and CD172 $\alpha^{\text {lo }}$ FSC $^{\text {hi }}$ (less mature) BM MP was analyzed (Figs. 5C and 5D).

The CD172 $\alpha^{\text {hi }}\left(\mathrm{FSC}^{\mathrm{lo}}\right)$ MP in noninfected piglets were predominantly $\mathrm{CD}^{+} 4^{+}(70 \%)$, $\mathrm{CD} 163^{-}(76 \%)$ and $\mathrm{MHCII}^{-}(70 \%)$. The $\mathrm{CD} 14^{+} \mathrm{MP}$ were increased 24 and $72 \mathrm{~h}$ after infection while the $\mathrm{CD}_{1}{ }^{-} \mathrm{MP}$ transiently decreased $24 \mathrm{~h}$ after infection and then returned to the initial counts $72 \mathrm{~h}$ after infection. Comparable changes occurred in the counts of CD163-expressing MP. The $\mathrm{CD} 163^{+} \mathrm{MP}$ were increased 24 and $72 \mathrm{~h}$ after infection while the $\mathrm{CD} 163^{-}$MP transiently decreased $24 \mathrm{~h}$ after infection and then returned to the initial counts $72 \mathrm{~h}$ after infection. With respect to 
(A)

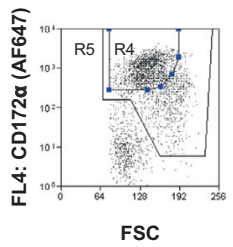

(C)
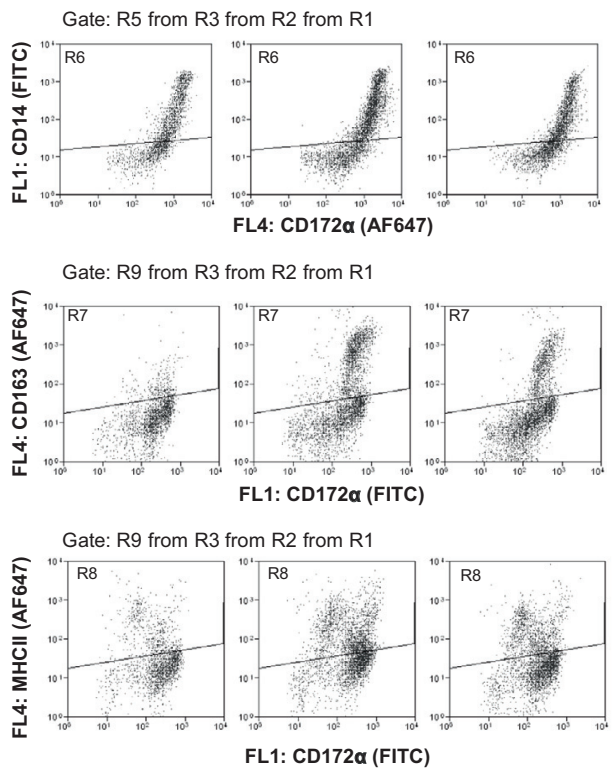

(E)

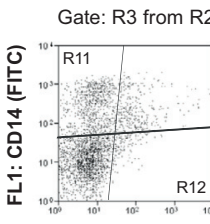

0
FL1: CD172 $\alpha$ (FITC)

\section{from R1}

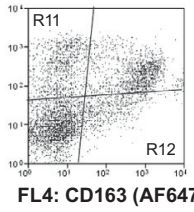

24

hours after infection
(B)

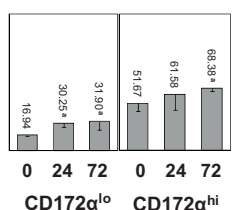

(D)
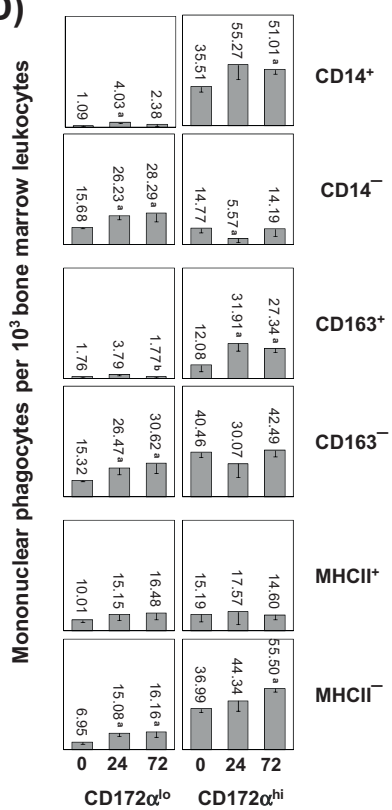

(F)

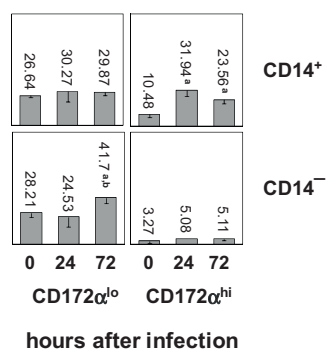

Figure 5. The cell surface molecule expression by bone marrow mononuclear phagocytes (MP) after Actinobacillus pleuropneumoniae (APP) infection. The gating of MP subpopulations was performed as described in Figure 2. Representative dot-plots of CD172 $\alpha$ (A), CD14, CD163 or MHCII versus CD172 $\alpha$ (C) and CD14 versus CD163 (E) expression by MP are shown. The absolute counts of MP expressing CD172 $\alpha$ (B), CD14, CD163 or MHCII versus CD172 $\alpha$ (D) and CD14 versus CD163 (F) per mL of bone marrow were calculated (data are mean \pm SEM). In the case of absolute counts of CD14 versus CD163 MP (F) the known counts of CD172 $\alpha^{\text {hi/lo }} \mathrm{CD} 14^{+/-}$and CD172 $\alpha^{\text {hi/lo }} \mathrm{CD} 163^{+/-}$(see Fig. 2F and Sect. 2), were used for calculations. Significant differences $(p<0.05)$ between noninfected $(0 \mathrm{~h}$ after infection) and APPinfected piglets are marked with "a". Significant differences $(p<0.05)$ between adjoining intervals are marked with " $b$ ". (A color version of this figure is available online at www.vetres.org.) 
MHCII expression, the $\mathrm{MHCII}^{-} \mathrm{MP}$ were increased after infection only, while $\mathrm{MHCII}^{+}$ MP remained unchanged.

Based on similar counts of the changed CD14 ${ }^{+/-}$CD163 $3^{+/-}$MP subpopulations, it could be assumed that those increased MP were CD14 ${ }^{+}$ and $\mathrm{CD}_{163^{+}}$and those transiently decreased MP were $\mathrm{CD}_{14}^{-}$and $\mathrm{CD} 163^{-}$. Similar to the situation for PB, however, this assumption could not be directly verified. The changes in absolute counts of $\mathrm{MHCII}^{-} \mathrm{MP}$ suggest that both these changed populations $\left(\mathrm{CD} 14^{+} \mathrm{CD} 163^{+}\right.$and $\mathrm{CD}^{-} 4^{-} \mathrm{CD}_{163^{-}}$) were $\mathrm{MHCII}^{-}$.

The CD172 $\alpha^{\text {lo }}\left(\mathrm{FSC}^{\mathrm{hi}}\right) \mathrm{MP}$ in noninfected piglets were predominantly $\mathrm{CD}^{-}(94 \%)$, $\mathrm{CD}^{-} 63^{-}(90 \%)$ and $\mathrm{MHCII}^{+}(59 \%)$. The MP that were increased after infection, were $\mathrm{CD}_{14}^{-}, \mathrm{CD}^{-} 63^{-}$and $\mathrm{MHCII}^{-}$. Since no changes occurred in the population of $\mathrm{CD} 14^{+}$ or $\mathrm{CD}_{163^{+}}$or $\mathrm{MHCII}^{+} \mathrm{MP}$, it could be assumed that these increased cells were $\mathrm{CD} 14^{-} \mathrm{CD} 163^{-}$ $\mathrm{MHCII}^{-}$.

To confirm the hypothesis that the MP population that changed after infection was $\mathrm{CD} 14^{+}$ $\mathrm{CD}_{163^{+}}$or $\mathrm{CD} 14^{-} \mathrm{CD} 163^{-}$, the CD14/CD163 (setup no. 4, Tab. II) staining and subsequent analysis was performed in the same way as in the PB. In noninfected piglets, two major $\left(\mathrm{CD} 14^{-} \mathrm{CD}^{-} 63^{-}\right.$switching into $\mathrm{CD} 14^{+}$ $\mathrm{CD} 63^{-}$) MP populations were found (Fig. 5E). A few cells only were $\mathrm{CD} 14^{+}$ $\mathrm{CD} 63^{+}$. After infection, there was a significant increase in $\mathrm{CD}_{14}^{+} \mathrm{CD}^{+} 63^{+}$. This population appeared to directly develop from $\mathrm{CD} 14^{-}$ $\mathrm{CD} 63^{-} \mathrm{BM} \mathrm{MP}$ rather than from $\mathrm{CD} 14^{+}$ $\mathrm{CD} 63^{-}$. Moreover, it was confirmed that the only populations that changed after infection were $\mathrm{CD} 14^{+} \mathrm{CD}_{163}{ }^{+}$or $\mathrm{CD} 14^{-} \mathrm{CD} 163^{-}$, while $\mathrm{CD}_{14}{ }^{+} \mathrm{CD}_{163^{-}}$and $\mathrm{CD} 14^{-} \mathrm{CD}^{-} 63^{+}$cells remained unchanged (Fig. 5F). The $\mathrm{CD} 14^{+}$ $\mathrm{CD}_{163^{+}} \mathrm{MP}$ were increased 24 as well as $72 \mathrm{~h}$ after infection. This represents the increased population of CD172 $\alpha^{\text {hi }}$ CD $14^{+} \mathrm{CD}_{163^{+}} \mathrm{MHCII}^{-}$ MP. The second population (i.e. $\mathrm{CD} 14^{-}$ CD163- MP) was increased $72 \mathrm{~h}$ after infection only (Fig. 5F). However, this lack of change after $24 \mathrm{~h}$ was probably due to the manner in which the data was presented. At this time point, two opposite trends could be detected: the numbers of CD172 $\alpha^{\text {lo }} \mathrm{CD} 14^{-} \mathrm{CD}^{-} 63^{-} \mathrm{MHCII}^{-}$were increased whereas the numbers of CD172 $\alpha^{\text {hi }}$ $\mathrm{CD}^{-}{ }^{-} \mathrm{CD} 163^{-} \mathrm{MHCII}^{-}$decreased (Fig. 5D), with the result that the sum of these two $\mathrm{CD}^{-} 4^{-} \mathrm{CD} 63^{-} \mathrm{MP}$ subpopulations remained unchanged $24 \mathrm{~h}$ after infection.

\section{DISCUSSION}

The aim of this work was to describe which porcine MP subpopulations infiltrate inflamed tissue under in vivo conditions. Moreover, the possible origin and maturation stages of these infiltrating MP were assessed in the PB and BM.

The cell surface molecules CD172 $\alpha$, SWC8 and CD203 $\alpha$ were used for the identification of basic MP populations (monocytes, dendritic cells, macrophages). The further discrimination of particular MP subpopulations was based on the presence of CD14, CD163 and MHCII molecules.

The initial identification of all MP was based on the detection of $\mathrm{CD} 172 \alpha$ together with SWC8 molecules according to Haverson et al. [12] and Summerfield et al. [19, 20]. In the $\mathrm{PB}$, two major populations of MP: the $\mathrm{CD} 172 \alpha^{\text {hi }} \mathrm{CD}^{+} 4^{+} \mathrm{SWC}^{-}$monocytes and CD172 $\alpha^{\text {lo }} \mathrm{CD}^{-} 4^{-} \mathrm{SWC}^{-}$dendritic cells [21] were identified. In the BM, typically two continuous subpopulations of MP were identified: less mature $\mathrm{CD} 172 \alpha^{\text {lo }} \mathrm{SWC}^{-} \mathrm{FSC}^{\text {hi }}$ and more mature $\mathrm{CD} 172 \alpha^{\text {hi }} \mathrm{SWC}^{-} \mathrm{FSC}^{\text {lo }}$ cells.

The CD203 $\alpha$ molecule was typically expressed on lung MP but not on PB or BM MP. Since this molecule appears during the development of monocytes into macrophages [14], the lung MP expressing the CD203 $\alpha$ molecule were considered to be more mature compared to those lacking this molecule.

After identification of basic MP populations, MP subpopulations were defined, based on the co-expression of other cell surface molecules. The expression profile of the CD14 molecule alone is well documented in the PB as well as in the BM [18-20]. Accordingly, CD14 was expressed by more mature (CD172 $\alpha^{\text {hi }} \mathrm{SWC}^{-}$ $\mathrm{FSC}^{\mathrm{lo}}$ ) but not by less mature (CD172 $\alpha^{\text {lo }}$ $\mathrm{SWC}^{-} \mathrm{FSC}^{\mathrm{hi}}$ ) BM MP and by PB monocytes, but not by dendritic cells. Thus, the use of the CD14 molecule alone for identifying MP 
subpopulations could yield only little new knowledge. However, the combination of this molecule with two other molecules (CD163 and MHCII) allows the discrimination of up to four distinct MP subpopulations [5, 15]. So far, the heterogeneity of the MP subpopulations has been described in the PB only using these three crucial surface molecules [5]. The present study provides new information about these subpopulations in the BM and lungs, including their possible developmental relationship to $\mathrm{PB}$, under either noninflammatory or inflammatory conditions.

The results of the present study have shown that two different PB and three different BM monocyte subpopulations play roles in the inflammatory response induced by APP. In the $\mathrm{PB}$, the population of $\left(\mathrm{CD} 172 \alpha^{\mathrm{hi}}\right) \mathrm{CD} 14^{+}$ $\mathrm{CD}_{163}{ }^{+} \mathrm{MHCII}^{-}$monocytes was increased even $6 \mathrm{~h}$ after infection and probably was a source of $\mathrm{CD}_{14}{ }^{+} \mathrm{CD}_{163^{+}}$monocytes for the inflamed lung tissue. The (CD172 $\left.\alpha^{\text {hi }}\right) \mathrm{CD} 14^{+}$ $\mathrm{CD} 63^{-} \mathrm{MHCII}^{-}$monocytes probably play a role in the initiation of an inflammatory response prior to the $\mathrm{CD} 14^{+} \mathrm{CD} 163^{+}$monocytes taking over the role of monocytes infiltrating the inflamed tissue.

In the $\mathrm{BM}$, the more mature $\mathrm{BM}$ monocytes $\left(\mathrm{CD} 172 \alpha^{\mathrm{hi}}\right) \mathrm{CD}^{+} 4^{+} \mathrm{CD}_{163^{+}} \mathrm{MHCII}^{-}$were increased and probably were a source of $\left(\mathrm{CD} 172 \alpha^{\mathrm{hi}}\right) \mathrm{CD} 4^{+} \mathrm{CD}_{163}{ }^{+} \mathrm{MHCII}^{-} \mathrm{PB}$ monocytes. The second population of more mature monocytes, (CD172 $\left.\alpha^{\text {hi }}\right) \mathrm{CD}^{+} 4^{+} \mathrm{CD} 163^{-}$ $\mathrm{MHCII}^{-}$, was transiently decreased $24 \mathrm{~h}$ after infection. This was probably caused by a sudden need for monocytes, which are the cells of sequential developmental stages within the BM or PB. The increased count of less matured cells, $\left(\mathrm{CD} 172 \alpha^{\mathrm{lo}}\right) \mathrm{CD}^{-} 4^{-} \mathrm{CD}^{-} 63^{-} \mathrm{MHCII}^{-}$, probably reflects the accelerated generation of the early monocyte precursors within the BM.

The population of $\mathrm{CD} 14^{+} \mathrm{CD} 163^{+} \mathrm{BM} \mathrm{MP}$ that was increased after infection appeared to develop directly from less mature $\mathrm{CD} 14^{-}$ $\mathrm{CD}_{163^{-}}$rather than from more mature $\mathrm{CD} 14^{+}$ CD163- BM MP. This could be caused by the accelerated upregulation of the CD163 molecule on the developing BM MP after receiving the inflammatory stimulus. These $\mathrm{CD} 14^{+} \mathrm{CD} 163^{+}$ $\mathrm{BM} \mathrm{MP}$ are then a direct source of PB monocytes during inflammation. However, this type of changed course of cell maturation is relatively unusual in biology. More common during enhanced consumption is the release of less mature cells from the BM to the PB (e.g. shift to the left in neutrophils, release of reticulocytes during regenerative anemia etc.). From this point of view, it is more probable that BM MP are produced by two different maturation pathways and then are released to the bloodstream as two distinct subpopulations. The use of these pathways differs under inflammatory and noninflammatory conditions.

This finding does not, however, exclude the possibility of CD163- $\mathrm{PB}$ monocytes switching into $\mathrm{CD} 63^{+}$monocytes, although this has so far been observed under in vitro conditions only [3]. It is likely that for a better understanding of the origin, differentiation pathways and function of MP in pigs, more comprehensive in vivo experiments are necessary in the future.

In the mouse model, two major subpopulations of PB monocytes exist: the Ly $6 \mathrm{C}^{+}$and Ly6C ${ }^{-}$cells. The phenotypic switch from Ly6C ${ }^{+}$into Ly6C $\mathrm{C}^{-}$monocytes is a spontaneous process occurring continuously in the bloodstream [23]. The less mature Ly6 $\mathrm{C}^{+}$monocytes could enter the inflamed tissue, while more mature Ly6 $\mathrm{C}^{-}$monocytes renew the population of resident macrophages [10, 13]. So far, very similar developmental pathways were suggested in pigs, where CD163- $\mathrm{PB}$ monocytes could under in vitro conditions spontaneously switch their phenotype to $\mathrm{CD}_{163}{ }^{+}$[5]. Since the population of PB MP that was increased under inflammatory conditions in the pig was $\mathrm{CD}_{163}{ }^{+}$ $\mathrm{CD} 14^{+}$, it could be suggested that, on the contrary to mice, the subpopulation of these more mature $\mathrm{CD} 163^{+} \mathrm{PB}$ monocytes plays a role in the infiltration of the inflamed tissue.

The combination of cell surface molecules used in the present study was found to be appropriate for characterizing MP subpopulations in pigs. However, an upgrade of the four-color into seven-color flow cytometry for simultaneous detection of all these molecules together seems to be necessary for future experiments.

From a functional point of view, the porcine $\mathrm{CD}_{163}{ }^{+} \mathrm{PB} \mathrm{MP}$ are known to express higher amounts of co-stimulatory and adhesion 
molecules than CD163- MP [3]. Moreover, they are able to produce higher amounts of TNF- $\alpha$ after in vitro cultivation [5]. Taken together, the $\mathrm{CD}_{163}{ }^{+} \mathrm{MP}$ exert a higher degree of preparedness to play a role in the defense against pathogenic agents compared to $\mathrm{CD} 163^{-}$MP. This closely corresponds to the present data demonstrating an important role for $\mathrm{CD} 163^{+} \mathrm{MP}$ during the inflammatory response.

An important role of CD163 $3^{+} \mathrm{PB} \mathrm{MP}$, which produce higher amounts of TNF- $\alpha$, is finally supported by the fact that TNF- $\alpha$ was found to play a role in the pathophysiology of APPinduced lung inflammation in pigs [6].

In summary, our results show that the $\mathrm{CD}_{14}{ }^{+} \mathrm{CD}_{163^{+}} \mathrm{MP}$ subpopulation is involved in infiltrating the inflamed tissue of pigs under in vivo conditions and that the initial response to the inflammatory stimulus appears to occur already in the bone marrow.

Acknowledgements. This study was supported by grants of the Czech Science Foundation (P502/10/ P362), Ministry of Agriculture of the Czech Republic (MZE0002716202) and Ministry of Education, Youth and Sports of the Czech Republic (CZ.1.05/2.1.00/ 01.0006, AdmireVet). The authors wish to thank Mrs. Ludmila Faldikova for critical reading of the manuscript.

\section{REFERENCES}

[1] Baarsch M.J., Foss D.L., Murtaugh M.P., Pathophysiologic correlates of acute porcine pleuropneumonia, Am. J. Vet. Res. (2000) 61:684-690.

[2] Bosse J.T., Janson H., Sheehan B.J., Beddek A.J., Rycroft A.N., Kroll J.S., Langford P.R., Actinobacillus pleuropneumoniae: pathobiology and pathogenesis of infection, Microbes Infect. (2002) 4:225-235.

[3] Chamorro S., Revilla C., Alvarez B., LopezFuertes L., Ezquerra A., Dominguez J., Phenotypic characterization of monocyte subpopulations in the pig, Immunobiology (2000) 202:82-93.

[4] Chamorro S., Revilla C., Gomez N., Alvarez B., Alonso F., Ezquerra A., Dominguez J., In vitro differentiation of porcine blood $\mathrm{CD}^{-} 63^{-}$and CD163 ${ }^{+}$monocytes into functional dendritic cells, Immunobiology (2004) 209:57-65.

[5] Chamorro S., Revilla C., Alvarez B., Alonso F., Ezquerra A., Dominguez J., Phenotypic and functional heterogeneity of porcine blood monocytes and its relation with maturation, Immunology (2005) 114: 63-71.

[6] Cho W.S., Chae C., Expression of nitric oxide synthase 2 and tumor necrosis factor $\alpha$ in swine naturally infected with Actinobacillus pleuropneumoniae, Vet. Pathol. (2002) 39:27-32.

[7] Delventhal S., Hensel A., Petzoldt K., Pabst R., Cellular changes in the bronchoalveolar lavage (BAL) of pigs, following immunization by the enteral or respiratory route, Clin. Exp. Immunol. (1992) 90:223227.

[8] Faldyna M., Nechvatalova K., Sinkora J., Knotigova P., Leva L., Krejci J., Toman M., Experimental Actinobacillus pleuropmeumoniae infection in piglets with different types and levels of specific protection: Immunophenotypic analysis of lymphocyte subsets in the circulation and respiratory mucosal lymphoid tissue, Vet. Immunol. Immunopathol. (2005) 107:143-152.

[9] Fogg D.K., Sibon C., Miled C., Jung S., Aucouturier P., Littman D.R., et al., A clonogenic bone marrow progenitor specific for macrophages and dendritic cells, Science (2006) 311:83-87.

[10] Geissmann F., Jung S., Littman D.R., Blood monocytes consist of two principal subsets with distinct migratory properties, Immunity (2003) 19:71-82.

[11] Gordon S., Taylor P.R., Monocyte and macrophage heterogeneity, Nat. Rev. Immunol. (2005) 5:953-964.

[12] Haverson K., Bailey M., Higgins V.R., Bland P.W., Stokes C.R., Characterization of monoclonal antibodies specific for monocytes, macrophages and granulocytes from porcine peripheral blood and mucosal tissues, J. Immunol. Methods (1994) 170:233-245.

[13] Landsman L., Jung S., Lung macrophages serve as obligatory intermediate between blood monocytes and alveolar macrophages, J. Immunol. (2007) 179: 3488-3494.

[14] McCullough K.C., Schaffner R., Natale V., Kim Y.B., Summerfield A., Phenotype of porcine monocytic cells: modulation of surface molecule expression upon monocyte differentiation into macrophages, Vet. Immunol. Immunopathol. (1997) 58:265-275.

[15] Sanchez C., Domenech N., Vazquez J., Alonso F., Ezquerra A., Dominguez J., The Porcine 2A10 antigen is homologous to human CD163 and related to macrophage differentiation, J. Immunol. (1999) 162: 5230-5237.

[16] Serbina N.V., Pamer E.G., Monocyte emigration from bone marrow during bacterial infection requires 
signals mediated by chemokine receptor CCR2, Nat. Immunol. (2006) 7:311-317.

[17] Strauss-Ayali D., Conrad S.M., Mosser D.M., Monocyte subpopulations and their differentiation patterns during infection, J. Leukoc. Biol. (2007) $82: 244-252$.

[18] Summerfield A., McCullough K., Porcine bone marrow myeloid cells: phenotype and adhesion molecule expression, J. Leukoc. Biol. (1997) 62: 176-185.

[19] Summerfield A., Haverson K., Thacker E., McCullough K.C., Differentiation of porcine myeloid bone marrow haematopoietic cell populations, Vet. Immunol. Immunopathol. (2001) 80:121-129.

[20] Summerfield A., Guzylack-Piriou L., Schaub A., Carrasco C.P., Tache V., Charley B., McCullough K.C., Porcine peripheral blood dendritic cells and natural interferon-producing cells, Immunology (2003) 110:440-449.
[21] Summerfield A., McCullough K.C., The porcine dendritic cell family, Dev. Comp. Immunol. (2009) 33:299-309.

[22] Sunderkotter C., Nikolic T., Dillon M.J., Van Rooijen N., Stehling M., Drevets D.A., Leenen P.J., Subpopulations of mouse blood monocytes differ in maturation stage and inflammatory response, J. Immunol. (2004) 172:4410-4417.

[23] Xu H., Manivannan A., Dawson R., Crane I.J., Mack M., Sharp P., Liversidge J., Differentiation to the $\mathrm{CCR} 2^{+}$inflammatory phenotype in vivo as a constitutive, time-limited property of blood monocytes and is independent of local inflammatory mediators, J. Immunol. (2005) 175:6915-6923.

[24] Zelnickova P., Faldyna M., Stepanova H., Ondracek J., Kovaru F., Intracellular cytokine detection by flow cytometry in pigs: fixation, permeabilization and cell surface staining, J. Immunol. Methods (2007) 327:18-29. 